\title{
A NOETHER-DEURING THEOREM FOR DERIVED CATEGORIES
}

\author{
ALEXANDER ZIMMERMANN \\ Université de Picardie, Département de Mathématiques et LAMFA (UMR 7352 du CNRS), \\ 33 rue St Leu, F-80039 Amiens Cedex 1, France \\ e-mail: alexander.zimmermann@u-picardie.fr
}

(Received 15 November 2011; revised 28 December 2011; accepted 3 January 2012; first published online 30 March 2012)

\begin{abstract}
We prove a Noether-Deuring theorem for the derived category of bounded complexes of modules over a Noetherian algebra.

2010 Mathematics Subject Classification. Primary 16E35; Secondary 11S36, 13J10, $18 \mathrm{E} 30,16 \mathrm{G} 30$.
\end{abstract}

1. Introduction. The classical Noether-Deuring theorem states that given an algebra $A$ over a field $K$ and a finite extension field $L$ of $K$, two $A$-modules $M$ and $N$ are isomorphic as $A$-modules, if $L \otimes_{K} M$ is isomorphic to $L \otimes_{K} N$ as an $L \otimes_{K} A$ module. In 1972, Roggenkamp gave a nice extension of this result to extensions $S$ of local commutative Noetherian rings $R$ and modules over Noetherian $R$-algebras.

For the derived category of $A$-modules no such generalisation was documented before. The purpose of this note is to give a version of the Noether-Deuring theorem, in the generalised version given by Roggenkamp, for right bounded derived categories of $A$-modules. If there is a morphism $\alpha \in \operatorname{Hom}_{D(\Lambda)}(X, Y)$, then it is fairly easy to show that for a faithfully flat ring extension $S$ over $R$ the fact that $i d_{S} \otimes \alpha$ is an isomorphism implies that $\alpha$ is an isomorphism. This is done in proposition (1). More delicate is the question if only an isomorphism in $\operatorname{Hom}_{D\left(S \otimes_{R} \Lambda\right)}\left(S \otimes_{R} X, S \otimes_{R} Y\right)$ is given. Then, we need further finiteness conditions on $\Lambda$ and on $R$ and proceed by completion of $R$ and then a classical going-down argument. This is done in theorem (4) and corollary (8).

For the notation concerning derived categories, we refer to Verdier [6]. In particular, $D(A)$ (resp $D^{-}(A)$, resp $D^{b}(A)$ ) denotes the derived category of complexes (resp. right bounded complexes, resp. bounded complexes) of finitely generated $A$-modules, $K^{-}(A-p r o j)\left(\right.$ resp. $K^{b}(A-p r o j)$, resp $\left.K^{-, b}(A-p r o j)\right)$ is the homotopy category of right bounded complexes (resp. bounded complexes, resp. right bounded complexes with bounded homology) of finitely generated projective $A$-modules. For a complex $Z$, we denote by $H_{i}(Z)$ the homology of $Z$ in degree $i$, and by $H(Z)$ the graded module given by the homology of $Z$.

2. The result. We start with an easy observation.

Proposition 1. Let $R$ be a commutative ring and let $\Lambda$ be an R-algebra. Let $S$ be a commutative faithfully flat $R$-algebra. Denote by $D(\Lambda)$ the derived category of complexes of finitely generated $\Lambda$-modules. Then, if there is $\alpha \in \operatorname{Hom}_{D(\Lambda)}(X, Y)$ so that $i d_{S} \otimes_{R}^{\mathbb{L}} \alpha \in \operatorname{Hom}_{D\left(S \otimes_{R} \Lambda\right)}\left(S \otimes_{R}^{\mathbb{L}} X, S \otimes_{R}^{\mathbb{L}} Y\right)$ is an isomorphism in $D\left(S \otimes_{R} \Lambda\right)$, then $\alpha$ is an isomorphism in $D(\Lambda)$. 
Proof. Let $Z$ be a complex in $D(\Lambda)$. Since $S$ is flat over $R$ the functor $S \otimes_{R}-$ : $R-\operatorname{Mod} \longrightarrow S-M o d$ is exact, and hence the left derived functor $S \otimes_{R}^{\mathbb{R}}-$ coincides with the ordinary tensor product functor $S \otimes_{R}-$. We can therefore work with the usual tensor product and a complex $Z$ of $\Lambda$-modules.

We claim that since $S$ is flat, $S \otimes_{R}-$ induces an isomorphism $S \otimes_{R} H(Z) \simeq$ $H\left(S \otimes_{R}^{\mathbb{L} Z} Z\right)$.

If $\partial_{Z}$ is the differential of $Z$, then

$$
0 \longrightarrow \operatorname{ker}\left(\partial_{Z}\right) \longrightarrow Z \stackrel{\partial_{Z}}{\longrightarrow} \operatorname{im}\left(\partial_{Z}\right) \longrightarrow 0
$$

is exact in the category of $\Lambda$-modules.

Since $S$ is flat,

$$
0 \longrightarrow S \otimes_{R} \operatorname{ker}\left(\partial_{Z}\right) \longrightarrow S \otimes_{R} Z \stackrel{i d_{S} \otimes_{R} \partial_{Z}}{\longrightarrow} S \otimes_{R} i m\left(\partial_{Z}\right) \longrightarrow 0
$$

is exact. Hence,

$$
\operatorname{ker}\left(i d_{S} \otimes_{R} \partial_{Z}\right)=S \otimes_{R} \operatorname{ker}\left(\partial_{Z}\right) \text { and } i m\left(i d_{S} \otimes_{R} \partial_{Z}\right)=S \otimes_{R} \operatorname{im}\left(\partial_{Z}\right) .
$$

This shows the claim.

Since $i d_{S} \otimes_{R} \alpha$ is an isomorphism, its cone $C\left(i d_{S} \otimes_{R} \alpha\right)$ is acyclic. Moreover, $C\left(i d_{S} \otimes_{R} \alpha\right)=S \otimes_{R} C(\alpha)$ by the very construction of the mapping cone. But now,

$$
0=H\left(C\left(i d_{S} \otimes_{R} \alpha\right)\right)=H\left(S \otimes_{R} C(\alpha)\right)=S \otimes_{R} H(C(\alpha)) .
$$

Since $S$ is faithfully flat, this implies $H(C(\alpha))=0$, and therefore, $C(\alpha)$ is acyclic. We conclude that $\alpha$ is an isomorphism in $D(\Lambda)$ which shows the statement.

REMARK 2. Observe that we assumed that $X \stackrel{\alpha}{\longrightarrow} Y$ is assumed to be a morphism in $D(\Lambda)$. The question if the existence of an isomorphism $S \otimes_{R} X \stackrel{\hat{\alpha}}{\longrightarrow} S \otimes_{R} Y$ in $D\left(S \otimes_{R} \Lambda\right)$ implies the existence of a morphism $\alpha: X \longrightarrow Y$ in $D(\Lambda)$ so that $i d_{S} \otimes_{R}^{\mathbb{L}} \alpha$ is an isomorphism is left open. Under stronger hypotheses, this is the purpose of Theorem 4 below. The proof follows [5] which deals with the module case.

LEMмA 3. If $S$ is a faithfully flat $R$-module and $\Lambda$ is a Noetherian R-algebra, then for all objects $X$ and $Y$ of $D^{b}(\Lambda)$, we get

$$
\operatorname{Hom}_{D^{b}\left(S \otimes_{R} \Lambda\right)}\left(S \otimes_{R} X, S \otimes_{R} Y\right) \simeq S \otimes_{R} \operatorname{Hom}_{D^{b}(\Lambda)}(X, Y) .
$$

Proof. Since $S$ is flat over $R$, the functor $S \otimes_{R}$ - preserves quasi-isomorphisms, and therefore, we get a morphism

$$
S \otimes_{R} \operatorname{Hom}_{D^{b}(\Lambda)}(U, V) \longrightarrow \operatorname{Hom}_{D^{b}\left(S \otimes_{R} \Lambda\right)}\left(S \otimes_{R} U, S \otimes_{R} V\right)
$$

in the following way. Given a morphism $\rho$ in $\operatorname{Hom}_{D^{b}(\Lambda)}(U, V)$ represented by the triple $(U \stackrel{\alpha}{\longleftarrow} W \stackrel{\beta}{\longrightarrow} V$ ), for a quasi-isomorphism $\alpha$ and a morphism of complexes $\beta$, and $s \in S$ then map $s \otimes \rho$ to $\left(S \otimes_{R} U \stackrel{i d_{S} \otimes \alpha}{\longleftarrow} S \otimes_{R} W \stackrel{s \otimes \beta}{\longrightarrow} S \otimes_{R} V\right)$. This is natural in $U$ and $V$.

We use the equivalence of categories $K^{-, b}(\Lambda-\operatorname{proj}) \simeq D^{b}(\Lambda)$ and suppose, therefore, that $X$ and $Y$ are right bounded complexes of finitely generated projective 
$\Lambda$-modules. But

$$
S \otimes_{R} \operatorname{Hom}_{\Lambda}\left(\Lambda^{n}, U\right)=S \otimes_{R} U^{n}=\left(S \otimes_{R} U\right)^{n}=H_{o m_{S} \Lambda}\left(\left(S \otimes_{R} \Lambda\right)^{n}, S \otimes_{R} U\right)
$$

which proves the statement in case $X$ or $Y$ is in $K^{b}(A-p r o j)$ since then a homomorphism is given by a direct sum of finitely many homogeneous mappings in those degrees where the complexes do both have non-zero components. Now, tensor product commutes with direct sums.

We come to the general case. Recall the so-called stupid truncation $\tau_{N}$ of a complex. Let $Z$ be a complex in $K^{-, b}(\Lambda-p r o j)$, denoted by $\partial$ its differential and let $N \in \mathbb{N}$ so that $H_{n}(Z)=0$ for all $n \geq N$. We denote the homogeneous components of $\partial$ so that $\partial_{n}: Z_{n} \longrightarrow Z_{n-1}$ for all $n$. Let $\tau_{N} Z$ be the complex given by $\left(\tau_{N} Z\right)_{n}=Z_{n}$ if $n \leq N$ and $\left(\tau_{N} Z\right)_{n}=0$ else. The differential $\delta$ on $\tau_{N} Z$ is defined to be $\delta_{n}=\partial_{n}$ if $n \leq N$ and $\delta_{n}=0$ else. Now, $\operatorname{ker}\left(\partial_{N}\right)=: C_{N}(Z)$ is a finitely generated $\Lambda$-module. Therefore, we get an exact triangle, called in the sequel the truncation triangle for $Z$,

$$
\tau_{N} Z \longrightarrow Z \longrightarrow C_{N}(Z)[N+1] \longrightarrow\left(\tau_{N} Z\right)[1]
$$

for all objects $Z$ in $K^{-, b}(A-p r o j)$. Obviously, $\tau_{N}\left(S \otimes_{R} Z\right)=S \otimes_{R} \tau_{N} Z$ and since $S$ is flat over $R$ also $C_{N}\left(S \otimes_{R} Z\right)=S \otimes_{R} C_{N}(Z)$.

We choose $N$ so that $H_{n}(X)=H_{n}(Y)=0$ for all $n \geq N$. To simplify the notation denote for the moment the bifunctor $\operatorname{Hom}_{K^{-, b}(\Lambda-p r o j)}(-,-)$ by $(-,-)$, the bifunctor $\operatorname{Hom}_{K^{-, b}\left(S \otimes_{R} \Lambda-p r o j\right)}(-,-)$ by $(-,-)_{S}$ and the bifunctor $S \otimes_{R} \operatorname{Hom}_{K^{-, b}(\Lambda-p r o j)}(-,-)$ by $S(-,-)$. Further, put $S \otimes_{R} X=: X_{S}$ and $S \otimes_{R} Y=: Y_{S}$. From the long exact sequence obtained by applying $\left(X_{S},-\right)_{S}$ to the truncation triangle of $Y_{S}$ we get a commutative diagram with exact lines $(\dagger)$

$$
\begin{aligned}
& \left(X_{S}, C_{N}\left(Y_{S}\right)[N]\right)_{S} \rightarrow\left(X_{S}, \tau_{N} Y_{S}\right)_{S} \rightarrow\left(X_{S}, Y_{S}\right)_{S} \rightarrow\left(X_{S}, C_{N}\left(Y_{S}\right)[N+1]\right)_{S} \rightarrow\left(X_{S}, \tau_{N} Y_{S}[1]\right)_{S}
\end{aligned}
$$

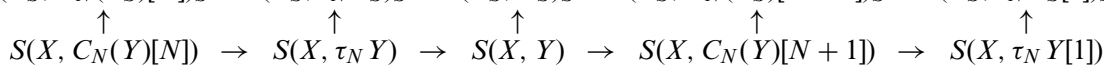

Since $\tau_{N}\left(Y_{S}\right)$ is a bounded complex of projectives,

$$
\left(X_{S}, \tau_{N} Y_{S}\right)_{S}=S \otimes_{R}\left(X, \tau_{N} Y\right) \text { and }\left(X_{S}, \tau_{N} Y_{S}[1]\right)_{S}=S \otimes_{R}\left(X, \tau_{N} Y[1]\right) .
$$

We apply $\left(-, C_{N}\left(Y_{S}\right)[N+1]\right)_{S}$ to the truncation triangle for $X_{S}$ and obtain an exact sequence

$$
\begin{aligned}
\left(\tau_{N} X_{S}[1], C_{N}\left(Y_{S}\right)[N+1]\right)_{S} & \rightarrow\left(C_{N}\left(X_{S}\right)[N+1], C_{N}\left(Y_{S}\right)[N+1]\right)_{S} \\
& \rightarrow\left(X_{S}, C_{N}\left(Y_{S}\right)[N+1]\right)_{S} \rightarrow\left(\tau_{N} X_{S}, C_{N} Y_{S}[N+1]\right)_{S} \\
& \rightarrow\left(C_{N}\left(X_{S}\right)[N], C_{N}\left(Y_{S}\right)[N+1]\right)_{S}
\end{aligned}
$$

and a commutative diagram analogous to the diagram $(\dagger)$. Now, for morphisms between finitely presented $\Lambda$-modules $M$ and $N$, we do have that the natural map

$$
S \otimes_{R} \operatorname{Hom}_{\Lambda}(M, N) \longrightarrow \operatorname{Hom}_{S \otimes_{R} \Lambda}\left(S \otimes_{R} M, S \otimes_{R} N\right)
$$

is an isomorphism (cf. [2, Proposition 2.10]). Given a projective resolution $P_{\bullet} \longrightarrow M$ of $M$, denote by $\partial_{n}: \Omega^{n} M \hookrightarrow P_{n-1}$ the embedding of the $n$-th syzygy of $M$ into the degree $n-1$ homogeneous component of the projective resolution. Then

$$
\operatorname{Ext}_{\Lambda}^{n}(M, N)=\operatorname{Hom}_{\Lambda}\left(\Omega^{n} M, N\right) /\left(\operatorname{Hom}_{\Lambda}\left(P_{n-1}, N\right) \circ \partial_{n}\right)
$$


and therefore,

$$
\begin{aligned}
S \otimes_{R} \operatorname{Ext}_{\Lambda}^{n}(M, N)= & S \otimes_{R}\left(\operatorname{Hom}_{\Lambda}\left(\Omega^{n} M, N\right) / \operatorname{Hom}_{\Lambda}\left(P_{n-1}, N\right) \circ \partial_{n}\right) \\
= & \left(S \otimes_{R} \operatorname{Hom}_{\Lambda}\left(\Omega^{n} M, N\right)\right) /\left(S \otimes_{R}\left(\operatorname{Hom}_{\Lambda}\left(P_{n-1}, N\right) \circ \partial_{n}\right)\right) \\
= & \operatorname{Hom}_{S \otimes_{R} \Lambda}\left(S \otimes_{R} \Omega^{n} M, S \otimes_{R} N\right) / \operatorname{Hom}_{S \otimes_{R} \Lambda}\left(S \otimes_{R} P_{n-1}, S \otimes_{R} N\right) \\
& \circ\left(1_{S} \otimes \partial_{n}\right) \\
= & \operatorname{Ext}_{S \otimes_{R} \Lambda}^{n}\left(S \otimes_{R} M, S \otimes_{R} N\right)
\end{aligned}
$$

for all $n \in \mathbb{N}$, natural in $M$ and $N$. This shows

$$
\left(C_{N}\left(X_{S}\right)[N+1], C_{N}\left(Y_{S}\right)[N+1]\right)_{S}=S \otimes_{R}\left(C_{N}(X)[N+1], C_{N}(Y)[N+1]\right)
$$

and

$$
\left(C_{N}\left(X_{S}\right)[N], C_{N}\left(Y_{S}\right)[N+1]\right)_{S}=S \otimes_{R}\left(C_{N}(X)[N], C_{N}(Y)[N+1]\right)
$$

By the case for bounded complex of projectives, we get that the natural morphism is an isomorphism for

$$
\left(\tau_{N} X_{S}[1], C_{N}\left(Y_{S}\right)[N+1]\right)_{S} \simeq S \otimes_{R}\left(\tau_{N} X[1], C_{N}(Y)[N+1]\right)
$$

and

$$
\left(\tau_{N} X_{S}, C_{N}\left(Y_{S}\right)[N+1]\right)_{S} \simeq S \otimes_{R}\left(\tau_{N} X, C_{N}(Y)[N+1]\right) .
$$

Therefore, also

$$
\left(X_{S}, C_{N}\left(Y_{S}\right)[N+1]\right)_{S} \simeq S \otimes_{R}\left(X, C_{N}(Y)[N+1]\right)
$$

and by the very same arguments

$$
\left(X_{S}, C_{N}\left(Y_{S}\right)[N]\right)_{S} \simeq S \otimes_{R}\left(X, C_{N}(Y)[N]\right) .
$$

This shows that, we get, isomorphisms in the two left and the two right vertical morphisms of $(\dagger)$ and hence also the central vertical morphism is an isomorphism. Hence

$$
\left(X_{S}, Y_{S}\right)_{S} \simeq S \otimes_{R}(X, Y)
$$

and the lemma is proved.

THEOREM 4. Let $R$ be a commutative Noetherian ring, let $S$ be a commutative Noetherian R-algebra and suppose that $S$ is a faithfully flat $R$-module. Suppose $S \otimes_{R}$ $\operatorname{rad}(R)=\operatorname{rad}(S)$. Let $\Lambda$ be a Noetherian $R$-algebra, let $X$ and $Y$ be two objects of of $D^{b}(\Lambda)$ and suppose that $\operatorname{End}_{D^{b}(\Lambda)}(X)$ is a finitely generated $R$-module. Then,

$$
S \otimes_{R}^{\mathbb{L}} X \simeq S \otimes_{R}^{\mathbb{Q}} Y \Leftrightarrow X \simeq Y .
$$

REMARK 5. We observe that, if $R$ is local and $S=\hat{R}$ is the $\operatorname{rad}(R)$-adic completion, then $S$ is faithfully flat as $R$-module and $S \otimes_{R} \operatorname{rad}(R)=\operatorname{rad}(S)$.

Proof of theorem 4. According to the hypotheses, we now suppose that $\operatorname{End}_{D^{b}(\Lambda)}(X)$ and $\operatorname{End}_{D^{-}(\Lambda)}(Y)$ are finitely generated $R$-module and that $S \otimes_{R} \operatorname{rad}(R)=\operatorname{rad}(S)$. Since 
$S$ is flat over $R$, tensor product of $S$ over $R$ is exact and we may replace the left derived tensor product by the ordinary tensor product. We only need to show " $\Rightarrow$ " and assume, therefore, that $X$ and $Y$ are in $K^{-, b}(\Lambda-$ proj $)$, and that $S \otimes_{R} X$ and $S \otimes_{R} Y$ are isomorphic.

Let $X_{S}:=S \otimes_{R} X$ and $S \otimes_{R} Y=: Y_{S}$ in $D^{b}\left(S \otimes_{R} \Lambda\right)$ to shorten the notation and denote by $\varphi_{S}$ the isomorphism $X_{S} \longrightarrow Y_{S}$. Since then $X_{S}$ is a direct factor of $Y_{S}$ by means of $\varphi_{S}$, the mapping

$$
\varphi_{S}=\sum_{i=1}^{n} s_{i} \otimes \varphi_{i}: X_{S} \longrightarrow Y_{S}
$$

for $s_{i} \in S$ and $\varphi_{i} \in \operatorname{Hom}_{D^{b}(\Lambda)}(X, Y)$ has a left inverse $\psi: Y_{S} \longrightarrow X_{S}$, so that,

$$
\psi \circ \varphi_{S}=i d_{X_{S}}
$$

Then,

$$
0 \longrightarrow \operatorname{rad}(R) \longrightarrow R \longrightarrow R / \operatorname{rad}(R) \longrightarrow 0
$$

is exact and since $S$ is flat over $R$, we get that

$$
0 \longrightarrow S \otimes_{R} \operatorname{rad}(R) \longrightarrow S \longrightarrow S \otimes_{R}(R / \operatorname{rad}(R)) \longrightarrow 0
$$

is exact. This shows that,

$$
S \otimes_{R}(R / \operatorname{rad}(R)) \simeq S /\left(S \otimes_{R} \operatorname{rad}(R)\right) .
$$

By hypothesis, we have $S \otimes_{R} \operatorname{rad}(R)=\operatorname{rad}(S)$, identifying canonically $S \otimes_{R} R \simeq S$. Then, there are $r_{i} \in R$ so that $1_{S} \otimes r_{i}-s_{i} \in \operatorname{rad}(S)$ for all $i \in\{1, \ldots, n\}$.

Put

$$
\varphi:=\sum_{i=1}^{n} r_{i} \varphi_{i} \in \operatorname{Hom}_{D^{b}(\Lambda)}(X, Y) .
$$

Then,

$$
\begin{aligned}
\sum_{i=1}^{n} \psi \circ\left(1_{S} \otimes\left(r_{i} \varphi_{i}\right)\right)-1_{S} \otimes i d_{X} & =\sum_{i=1}^{n}\left(\psi \circ\left(1_{S} \otimes r_{i} \varphi_{i}\right)-\psi \circ\left(s_{i} \otimes \varphi_{i}\right)\right) \\
& =\sum_{i=1}^{n}\left(1_{S} \otimes r_{i}-s_{i}\right) \cdot\left(\psi \circ\left(i d_{S} \otimes \varphi_{i}\right)\right) \\
& \in\left(\operatorname{rad}(S) \otimes_{R} \operatorname{End}_{D^{b}(\Lambda)}(X)\right)
\end{aligned}
$$

and since $E n d_{D^{b}(\Lambda)}(X)$ is a Noetherian $R$-module, using Nakayama's lemma, we obtain that $\psi \circ\left(\sum_{i=1}^{n} 1_{S} \otimes r_{i} \varphi_{i}\right)$ is invertible in $S \otimes_{R} E n d_{D^{b}(\Lambda)}(X)$. Hence, $i d_{S} \otimes_{R} \varphi$ is left split, and therefore,

$$
X_{S} \stackrel{i d_{S} \otimes_{R} \varphi}{\longrightarrow} Y_{S} \longrightarrow C\left(i d_{S} \otimes_{R} \varphi\right) \stackrel{0}{\longrightarrow} X_{S}[1]
$$

is a distinguished triangle, with $C\left(i d_{S} \otimes_{R} \varphi\right)$ being the cone of $i d_{S} \otimes_{R} \varphi$. However,

$$
C\left(i d_{S} \otimes_{R} \varphi\right)=S \otimes_{R} C(\varphi)
$$


and hence,

$$
X_{S} \stackrel{i d_{S} \otimes_{R} \varphi}{\longrightarrow} Y_{S} \longrightarrow S \otimes_{R} C(\varphi) \stackrel{0}{\longrightarrow} X_{S}[1]
$$

is a distinguished triangle.

Since $\varphi_{S}$ is an isomorphism, $\varphi_{S}$ has a right inverse $\chi: Y_{S} \longrightarrow X_{S}$ as well. Now, since $X_{S} \simeq Y_{S}, S$ is faithfully flat over $R$, and $\operatorname{End}_{D^{b}(\Lambda)}(X)$ is finitely generated as $R$ module; using Lemma 3, we obtain that $\operatorname{End}_{D^{b}(\Lambda)}(Y)$ is finitely generated as $R$-module as well. The same argument as for the left inverse $\psi$ shows that $\left(i d_{S} \otimes \varphi\right) \circ \chi$ is invertible in $S \otimes_{R} \operatorname{End}_{D^{b}(\Lambda)}(Y)$. Hence,

$$
X_{S} \stackrel{i d_{S} \otimes_{R} \varphi}{\longrightarrow} Y_{S} \stackrel{0}{\longrightarrow} S \otimes_{R} C(\varphi) \stackrel{0}{\longrightarrow} X_{S}[1]
$$

is a distinguished triangle. This shows that $S \otimes_{R} C(\varphi)$ is acyclic, and hence,

$$
0=H\left(S \otimes_{R} C(\varphi)\right)=S \otimes_{R} H(C(\varphi))
$$

Since $S$ is faithfully flat over $R$ also $H(C(\varphi))=0$, which implies that $C(\varphi)$ is acyclic, and therefore, $\varphi$ is an isomorphism.

This proves the theorem.

Let $A$ be an algebra over a complete discrete valuation ring $R$ which is finitely generated as a module over $R$. We shall need a Krull-Schmidt theorem for the derived category of bounded complexes over $A$. This fact seems to be well-known, but for the convenience of the reader we give a proof.

Proposition 6. Let $R$ be a complete discrete valuation ring and let $A$ be an $R$ algebra, finitely generated as R-module. Then, the Krull-Schmidt theorem holds for $K^{-, b}(A-p r o j)$.

Proof. We first show a Fitting lemma for $K^{-, b}(A-$ proj $)$.

Let $X$ be a complex in $K^{-, b}(A-p r o j)$ and let $u$ be an endomorphism of the complex $X$. Then, $X=X^{\prime} \oplus X^{\prime \prime}$ as graded modules, by Fitting's lemma in the version for algebras over complete discrete valuation rings [1, Lemma 1.9.2]. The restriction of $u$ on $X^{\prime}$ is an automorphism in each degree and the restriction of $u$ on $X^{\prime \prime}$ is nilpotent modulo $\operatorname{rad}(R)^{m}$ for each $m$. Therefore, $u$ is a diagonal matrix $\left(\begin{array}{ll}c & 0 \\ 0 & v\end{array}\right)$ in each degree where $\iota: X^{\prime} \longrightarrow X^{\prime}$ is invertible, and $v: X^{\prime \prime} \longrightarrow X^{\prime \prime}$ is nilpotent modulo $\operatorname{rad}(R)^{m}$ for each $m$ in each degree. The differential $\partial$ on $X$ is given by $\left(\begin{array}{ll}\partial_{1} & \partial_{2} \\ \partial_{3} & \partial_{4}\end{array}\right)$ and the fact that $u$ commutes with $\partial$ shows that $\partial_{3} \iota=v \partial_{3}$ and $\partial_{2} v=\iota \partial_{2}$. Therefore, $\partial_{3} \iota^{s}=v^{s} \partial_{3}$ and $\partial_{2} v^{s}=\iota^{s} \partial_{2}$ for all $s$. Since $v$ is nilpotent modulo $\operatorname{rad}(R)^{m}$ for each $m$ in each degree, and $\iota$ is invertible, $\partial_{2}=\partial_{3}=0$. Hence, the differential of $X$ restricts to a differential on $X^{\prime}$ and a differential on $X^{\prime \prime}$. Moreover, $X^{\prime}$ and $X^{\prime \prime}$ are both projective modules, since $X$ is projective.

Now, $X$, and therefore, also $X^{\prime \prime}$ is exact in degrees higher than $N$, say. We fix $m \in \mathbb{N}$ and obtain, therefore, that $u$ is nilpotent modulo $\operatorname{rad}(R)^{m}$ in each degree lower than $N$. Let $M_{m}$ be the nilpotency degree. Then, since $X^{\prime \prime}$ is exact in degrees higher than $N$, modulo $\operatorname{rad}(R)^{m}$ the restriction of the endomorphism $u^{M_{m}}$ to $X^{\prime \prime}$ is homotopy equivalent to 0 in degrees higher than $N$. We get, therefore, that the restriction of $u$ to $X^{\prime \prime}$ is actually nilpotent modulo $\operatorname{rad}(R)^{m}$ for each $m$. 
Hence, the endomorphism ring of an indecomposable object is local and the Krull-Schmidt theorem is an easy consequence by the classical proof as in [4] or in [1].

This shows the proposition.

REMARK 7. If $R$ is a field and $A$ is a finite dimensional $R$-algebra, then, we would be able to argue more directly. Indeed, $X^{\prime}=\operatorname{im}\left(u^{N}\right)$ and $X^{\prime \prime}=\operatorname{ker}\left(u^{N}\right)$ for large enough $N$. Then, it is obvious that $X^{\prime}$ and $X^{\prime \prime}$ are both subcomplexes of $X$. Observe that $R$ may be a field in proposition 6 .

For the next Corollary, we follow closely [5].

COROllary 8. Let $R$ be a commutative semilocal Noetherian ring, let $S$ be a commutative $R$-algebra so that $\hat{S}:=\hat{R} \otimes_{R} S$ is a faithful projective $\hat{R}$-module of finite type. Let $\Lambda$ be a Noetherian $R$-algebra, finitely generated as $R$-module, and let $X$ and $Y$ be two objects of $D^{b}(\Lambda)$ and suppose that $\operatorname{End}_{D^{b}(\Lambda)}(X)$ and $\operatorname{End}_{D^{b}(\Lambda)}(Y)$ are finitely generated $R$-module. Then,

$$
S \otimes_{R}^{\mathbb{L}} X \simeq S \otimes_{R}^{\mathbb{L}} Y \Leftrightarrow X \simeq Y .
$$

Proof. If $S \otimes_{R}^{\mathbb{L}} X \simeq S \otimes_{R}^{\mathbb{L}} Y$ in $D^{b}\left(S \otimes_{R} \Lambda\right)$, we get $\hat{S} \otimes_{R}^{\mathbb{L}} X \simeq \hat{S} \otimes_{R}^{\mathbb{L}} Y$ in $D^{b}\left(\hat{S} \otimes_{R}\right.$ $\Lambda)$. Since $R$ is semilocal with maximal ideals $m_{1}, \ldots, m_{s}$, we get $\hat{R}=\prod_{i=1}^{s} \hat{R}_{m_{i}}$ for the completion $\hat{R}_{m_{i}}$ of $R$ at $m_{i}$. Now, $\hat{S}$ is projective faithful of finite type, and so, there are $n_{1}, \ldots, n_{s}$ with

$$
\hat{S} \simeq \prod_{i=1}^{s}\left(\hat{R}_{m_{i}}\right)^{n_{i}}
$$

and therefore, $\hat{S} \otimes_{R}^{\llbracket} X \simeq \hat{S} \otimes_{R}^{\llbracket} Y$ implies

$$
\prod_{i=1}^{s}\left(\hat{R}_{m_{i}}\right)^{n_{i}} \otimes_{R}^{\mathbb{Q}} X \simeq \prod_{i=1}^{s}\left(\hat{R}_{m_{i}}\right)^{n_{i}} \otimes_{R}^{\mathbb{Q}} Y .
$$

Hence,

$$
\left(\hat{R}_{m_{i}} \otimes_{R}^{\mathbb{L}} X\right)^{n_{i}} \simeq\left(\hat{R}_{m_{i}} \otimes_{R}^{\mathbb{L}} Y\right)^{n_{i}}
$$

for each $i$, and therefore by Proposition 6

$$
\hat{R}_{m_{i}} \otimes_{R}^{\mathbb{L}} X \simeq \hat{R}_{m_{i}} \otimes_{R}^{\mathbb{L}} Y
$$

for each $i$. By Theorem 4, we obtain $X \simeq Y$.

We get cancellation of factors from this statement.

COROLlary 9. Under the hypothesis of theorem 4 or of corollary 8 , we get $X \oplus U \simeq$ $Y \oplus U$ in $D^{b}(\Lambda)$ implies $X \simeq Y$.

Proof. This is clear by corollary 8 in combination with proposition 6 .

REMARK 10. In [3], we developed a theory to roughly speaking parameterise geometrically objects in $D^{b}(A)$ by orbits of a group action on a variety. For this purpose, we need to assume that $A$ is a finite dimensional algebra over an algebraically closed field $K$, so that it is possible to use arguments and constructions from algebraic 
geometry. Using theorem 4, we can extend the theory to non algebraically closed fields $K$ as well.

ACKNOWLEDGEMENT. I wish to thank the referee for useful suggestions which lead to substantial improvements.

\section{REFERENCES}

1. D. Benson, Representations and Cohomology I (Cambridge University Press, Cambridge, 1997).

2. D. Eisenbud, Commutative algebra, with a view toward algebraic geometry (Springer, New York, 2004).

3. B. T. Jensen, X. Su and A. Zimmermann, Degenerations for derived categories, J. Pure Appl. Algebra. 198 (2005), 281-295

4. S. Lang, Algebra (Addison-Wesley, Menlo Park California, 1993).

5. K. W. Roggenkamp, An extension of the Noether-Deuring theorem, Proc. Am. Math. Soc. 31 (1972), 423-426.

6. J.-L. Verdier, Des catégories dérivées des catégories abéliennes, Astérisque. 239 (1996). 